\title{
A COMPUTATION OF BUCHSBAUM-RIM FUNCTIONS OF TWO VARIABLES IN A SPECIAL CASE
}

\author{
FUTOSHI HAYASAKA
}

\begin{abstract}
In this paper, we will compute BuchsbaumRim functions of two variables associated to a parameter matrix of a special form over a one-dimensional CohenMacaulay local ring, and we will determine when the function coincides with the Buchsbaum-Rim polynomial. As a consequence, we have that there exists the case where the function does not coincide with the polynomial function, which should be contrasted with the ordinary BuchsbaumRim function of single variable.
\end{abstract}

1. Introduction. Let $(R, \mathfrak{m})$ be a Noetherian local ring with the maximal ideal $\mathfrak{m}$ of dimension $d>0$, and let $C$ be a nonzero $R$-module of finite length. Let $\varphi: R^{n} \rightarrow R^{r}$ be an $R$-linear map of free modules with $C=\operatorname{Coker} \varphi$ as the cokernel of $\varphi$, and set $M:=\operatorname{Im} \varphi \subset F:=R^{r}$. Then one may consider the function,

$$
\lambda(p):=\ell_{R}\left(\left[\operatorname{Coker} \operatorname{Sym}_{R}(\varphi)\right]_{p}\right)=\ell_{R}\left(S_{p} / M^{p}\right),
$$

where $S_{p}$ (respectively $M^{p}$ ) is a homogeneous component of degree $p$ of $S=\operatorname{Sym}_{R}(F)$ (respectively $R[M]=\operatorname{Im}_{\operatorname{Sym}_{R}}(\varphi)$ ). Buchsbaum-Rim [3] first introduced and studied this type of function and proved that $\lambda(p)$ is eventually a polynomial of degree $d+r-1$, which we call the Buchsbaum-Rim polynomial. Then they defined a multiplicity of $C$ as $e(C):=\left(\right.$ The coefficient of $p^{d+r-1}$ in the polynomial $) \times(d+r-1) !$,

2010 AMS Mathematics subject classification. Primary 13H15, Secondary $13 \mathrm{H} 10$.

Keywords and phrases. Buchsbaum-Rim multiplicity, parameter matrix, CohenMacaulay.

The author was partially supported by a JSPS Grant-in-Aid for Young Scientists, grant No. (B) 24740032.

Received by the editors on June 5, 2014, and in revised form on November 18, 2014. 
which we now call the Buchsbaum-Rim multiplicity of $C$. They also proved that the multiplicity is independent of the choice of $\varphi$. The multiplicity $e(C)$ coincides with the ordinary Hilbert-Samuel multiplicity of an ideal $I$ when $C$ is a cyclic module $R / I$.

Buchsbaum and Rim also introduced the notion of a parameter matrix, which generalizes the notion of a system of parameters. A matrix (a linear map of free modules) $\varphi$ over $R$ of size $r \times n$ (from $R^{n}$ to $R^{r}$ ) is said to be a parameter matrix for $R$, if the following three conditions are satisfied:

(i) Coker $\varphi$ has finite length,

(ii) $d=n-r+1$,

(iii) $\operatorname{Im} \varphi \subset \mathfrak{m} R^{r}$.

Then it is known that, if $R$ is Cohen-Macaulay and $\varphi$ is a parameter matrix, then there exist formulas

$$
e(C)=\ell_{R}(C)=\ell_{R}\left(R / \operatorname{Fitt}_{0}(C)\right)
$$

for the Buchsbaum-Rim multiplicity $[\mathbf{2}, \mathbf{3}, \mathbf{6}]$ and for the BuchsbaumRim function [1],

$$
\lambda(p)=e(C)\left(\begin{array}{c}
p+d+r-2 \\
d+r-1
\end{array}\right),
$$

for all $p \geq 0$. It is also known [5] that, for any $p \geq 0$, the inequality,

$$
\lambda(p) \geq e(C)\left(\begin{array}{c}
p+d+r-2 \\
d+r-1
\end{array}\right),
$$

always holds true for a parameter matrix $\varphi$ even if $R$ is not CohenMacaulay, and the equality for some $p>0$ characterizes the CohenMacaulay property of the ring $R$.

Kleiman and Thorup $[\mathbf{9}, \mathbf{1 0}]$ and Kirby and Rees $[\mathbf{7}, \mathbf{8}]$ introduced another kind of multiplicity associated to $C$, which is related to the Buchsbaum-Rim multiplicity (see also [4]). They considered the function of two variables,

$$
\Lambda(p, q):=\ell_{R}\left(S_{p+q} / M^{p} S_{q}\right),
$$

and proved that $\Lambda(p, q)$ is eventually a polynomial of total degree $d+r-1$. Then they defined a sequence of multiplicities, for $j=$ 


$$
\begin{aligned}
0,1, \ldots, d+r-1 & \\
e^{j}(C):= & \text { The coefficient of } \left.p^{d+r-1-j} q^{j} \text { in the polynomial }\right) \\
& \times(d+r-1-j) ! j !
\end{aligned}
$$

and proved that $e^{j}(C)$ is independent of the choice of $\varphi$. Moreover, they proved that

$$
e(C)=e^{0}(C) \geq e^{1}(C) \geq \cdots \geq e^{r-1}(C)>e^{r}(C)=\cdots=e^{d+r-1}(C)=0,
$$

where $r=\mu_{R}(C)$ is the minimal number of generators of $C$. Thus, we call $e^{j}(C)$ the $j$ th Buchsbaum-Rim multiplicity of $C$. Then it is natural to ask the following.

Problem 1.1. Let $\varphi: R^{n} \rightarrow R^{r}$ be a parameter matrix with $C=$ Coker $\varphi$. Suppose that $R$ is Cohen-Macaulay. Then:

(i) does there exist a simple formula for the Buchsbaum-Rim multiplicities $e^{j}(C)$ for $j=1,2, \ldots, r-1$ ?

(ii) Does the function $\Lambda(p, q)$ coincide with a polynomial function?

In this paper, we will try to calculate the function $\Lambda(p, q)$ and multiplicities $e^{j}(C)$ in a special case where $C$ is a direct sum of cyclic modules $R / Q_{i}$ and $Q_{i}$ is a parameter ideal in a one dimensional CohenMacaulay local ring $R$. In particular, in the case $C=R / Q_{1} \oplus R / Q_{2}$, we will determine when the function $\Lambda(p, q)$ coincides with a polynomial function (Theorem 2.4). As a consequence, we have that there exists the case where the function $\Lambda(p, q)$ does not coincide with a polynomial function. This should be contrasted with a result of Brennan, Ulrich and Vasconcelos [1] as stated above: the ordinary Buchsbaum-Rim function $\lambda(p)=\Lambda(p, 0)$ coincides with the Buchsbaum-Rim polynomial for all $p \geq 0$ in the case where $R$ is Cohen-Macaulay and $\varphi$ is a parameter matrix.

2. Computation in a special case. In what follows, let $(R, \mathfrak{m})$ be a one dimensional Cohen-Macaulay local ring with the maximal ideal $\mathfrak{m}$. Let $r>0$ be a fixed positive integer, and let $Q_{1}, Q_{2}, \ldots, Q_{r}$ be parameter ideals in $R$ with $Q_{i}=\left(x_{i}\right)$ for $i=1,2, \ldots, r$. We set $a_{i}=\ell_{R}\left(R / Q_{i}\right)=e\left(R / Q_{i}\right)$ for $i=1,2, \ldots, r$. Let $\varphi: R^{r} \rightarrow R^{r}$ be an 
$R$-linear map represented by a parameter matrix,

$$
\left(\begin{array}{cccc}
x_{1} & 0 & \cdots & 0 \\
0 & x_{2} & \ddots & \vdots \\
\vdots & \ddots & \ddots & 0 \\
0 & \ldots & 0 & x_{r}
\end{array}\right),
$$

and let $C=$ Coker $\varphi$, which is a direct sum $R / Q_{1} \oplus R / Q_{2} \oplus \cdots \oplus R / Q_{r}$ of cyclic modules. Let $S=\operatorname{Sym}_{R}\left(R^{r}\right)$ be the symmetric algebra of $R^{r}$, and let $N=\operatorname{Im} \varphi$ be the image of $\varphi$. With this notation, we want to compute the following:

- the function $\Lambda(p, q)=\ell_{R}\left(S_{p+q} / N^{p} S_{q}\right)$ for $p, q \geq 0$,

- the polynomial $\Lambda(p, q)=\ell_{R}\left(S_{p+q} / N^{p} S_{q}\right)$ for $p, q \gg 0$,

- the multiplicities $e^{j}(C)$ for $j=1,2, \ldots, r-1$.

In order to calculate the above, we fix a free basis $\left\{t_{1}, t_{2}, \ldots, t_{r}\right\}$ for $R^{r}$. Then $S=R\left[t_{1}, t_{2}, \ldots, t_{r}\right]$ is a polynomial ring and $N=$ $Q_{1} t_{1}+Q_{2} t_{2}+\cdots+Q_{r} t_{r} \subset S_{1}=R t_{1}+R t_{2}+\cdots+R t_{r}$. Thus, for any $p, q \geq 0$, the module $N^{p} S_{q}$ can be described as follows:

$$
\begin{aligned}
N^{p} S_{q} & =\left(\sum_{\substack{|\boldsymbol{j}|=p \\
\boldsymbol{j} \geq \mathbf{0}}} \boldsymbol{Q}^{\boldsymbol{j}} \boldsymbol{t}^{\boldsymbol{j}}\right)\left(\sum_{\substack{|\boldsymbol{k}|=q \\
\boldsymbol{k} \geq \mathbf{0}}} R \boldsymbol{t}^{\boldsymbol{k}}\right) \\
& =\sum_{\substack{|\ell|=p+q \\
\boldsymbol{\ell} \geq \mathbf{0}}}\left(\sum_{\substack{|\boldsymbol{k}|=q \\
\mathbf{0} \leq \boldsymbol{k} \leq \boldsymbol{\ell}}} \boldsymbol{Q}^{\boldsymbol{\ell}-\boldsymbol{k}}\right) \boldsymbol{t}^{\boldsymbol{\ell}} \subset S_{p+q} \\
& =\sum_{\substack{|\ell|=p+q \\
\boldsymbol{\ell} \geq \mathbf{0}}} R \boldsymbol{t}^{\boldsymbol{\ell}} .
\end{aligned}
$$

Here we use the multi-index notation: for a vector $\boldsymbol{i}=\left(i_{1}, \ldots, i_{r}\right) \in$ $\mathbb{Z}_{\geq 0}^{r}$, we denote $\boldsymbol{Q}^{\boldsymbol{i}}=Q_{1}^{i_{1}} \cdots Q_{r}^{i_{r}}, \boldsymbol{t}^{\boldsymbol{i}}=t_{1}^{i_{1}} \cdots t_{r}^{i_{r}}$ and $|\boldsymbol{i}|=i_{1}+\cdots+i_{r}$. For any vector $\ell=\left(\ell_{1}, \ldots, \ell_{r}\right) \in \mathbb{Z}_{\geq 0}^{r}$ such that $|\ell|=p+q$, we define the ideal in $R$ as follows:

$$
J_{p, q}(\ell):=\sum_{\substack{|\boldsymbol{k}|=q \\ \mathbf{0} \leq \boldsymbol{k} \leq \boldsymbol{\ell}}} Q^{\boldsymbol{\ell}-\boldsymbol{k}}=\sum_{\substack{|\boldsymbol{i}|=p \\ \mathbf{0} \leq \boldsymbol{i} \leq \boldsymbol{\ell}}} \boldsymbol{Q}^{\boldsymbol{i}}
$$


Then the function $\Lambda(p, q)$ can be described as

$$
\Lambda(p, q)=\ell_{R}\left(S_{p+q} / N^{p} S_{q}\right)=\sum_{\substack{|\ell|=p+q \\ \ell \geq \mathbf{0}}} \ell_{R}\left(R / J_{p, q}(\ell)\right)
$$

for any $p, q \geq 0$. Thus, in order to compute the function $\Lambda(p, q)$, it is enough to compute the colength $\ell_{R}\left(R / J_{p, q}(\ell)\right)$ of the ideal $J_{p, q}(\ell)$.

We first consider the special case where the set of ideals $Q_{1}, Q_{2}, \ldots, Q_{r}$ is an ascending chain. The function $\Lambda(p, q)$ can be explicitly computed as follows in this case.

Proposition 2.1. Suppose that $Q_{1} \subseteq Q_{2} \subseteq \cdots \subseteq Q_{r}$. Then

$$
\begin{aligned}
\Lambda(p, q)= & \left(a_{1}+\cdots+a_{r}\right)\left(\begin{array}{c}
p+r-1 \\
r
\end{array}\right) \\
& +\sum_{i=1}^{r-1}\left(a_{i+1}+\cdots+a_{r}\right)\left(\begin{array}{c}
p+r-i-1 \\
r-i
\end{array}\right)\left(\begin{array}{c}
q+i-1 \\
i
\end{array}\right)
\end{aligned}
$$

for all $p, q \geq 0$, where $\left(\begin{array}{c}m \\ n\end{array}\right)=0$ if $m<n$. In particular, the function $\Lambda(p, q)$ coincides with a polynomial function and

$$
e^{j}(C)= \begin{cases}a_{j+1}+\cdots+a_{r} & (j=0,1, \ldots, r-1) \\ 0 & (j=r)\end{cases}
$$

Proof. Let us fix any $p, q \geq 0$. The case $r=1$ is a well-known result for the Hilbert-Samuel function. The case $q=0$ is a result of $[\mathbf{1}$, Theorem 3.4] on the ordinary Buchsbaum-Rim function $\lambda(p)=\Lambda(p, 0)$. So we may assume that $r \geq 2$ and $q \geq 1$. Suppose $Q_{1} \subseteq Q_{2} \subseteq \cdots \subseteq Q_{r}$. Then the ideal $J_{p, q}(\ell)$ coincides with the ideal of the product of last $p$ ideals of a sequence of ideals

$$
\underbrace{\overbrace{Q_{1}, \ldots, Q_{1}}^{\ell_{1}}, \overbrace{Q_{2}, \ldots, Q_{2}}^{\ell_{2}}, \ldots, \overbrace{Q_{r}, \ldots, Q_{r}}^{\ell_{r}}}_{p+q} .
$$


Hence its colength $\ell_{R}\left(R / J_{p, q}(\ell)\right)$ is the sum of last $p$ integers of a sequence of integers

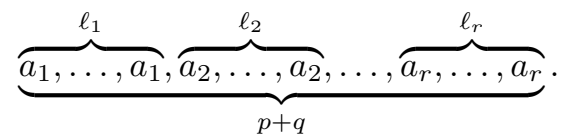

To compute the sum

$$
\sum_{\substack{|\ell|=p+q \\ \ell \geq \mathbf{0}}} \ell_{R}\left(R / J_{p, q}(\ell)\right)
$$

we divide the sequence $(2.1)$ at the $(p+1)$ th integer from the end. If the $(p+1)$ th integer from the end is $a_{i}$, then the sum of all last $p$ integers of such sequences can be counted by

$$
\left(\begin{array}{c}
(q-1)+i-1 \\
i-1
\end{array}\right)\left(\sum_{\substack{u_{i}+\cdots+u_{r}=p \\
u_{i}, \ldots, u_{r} \geq 0}}\left(u_{i} a_{i}+u_{i+1} a_{i+1}+\cdots+u_{r} a_{r}\right)\right) .
$$

Therefore,

$$
\begin{aligned}
& \Lambda(p, q)=\sum_{\substack{|\ell|=p+q \\
\ell \geq 0}} \ell_{R}\left(R / J_{p, q}(\ell)\right) \\
& =\sum_{i=1}^{r}\left(\begin{array}{c}
(q-1)+i-1 \\
i-1
\end{array}\right)\left(\sum_{\substack{u_{i}+\cdots+u_{r}=p \\
u_{i}, \ldots, u_{r} \geq 0}}\left(u_{i} a_{i}+u_{i+1} a_{i+1}+\cdots+u_{r} a_{r}\right)\right) \\
& =\sum_{i=1}^{r}\left(\begin{array}{c}
q+i-2 \\
i-1
\end{array}\right)\left(a_{i}+\cdots+a_{r}\right)\left(\begin{array}{c}
(r-i+1)+p-1 \\
p
\end{array}\right) \frac{p}{r-i+1} \\
& =\sum_{i=1}^{r}\left(a_{i}+\cdots+a_{r}\right)\left(\begin{array}{c}
q+i-2 \\
i-1
\end{array}\right)\left(\begin{array}{c}
r-i+p \\
p
\end{array}\right) \frac{p}{r-i+1} \\
& =\sum_{i=1}^{r}\left(a_{i}+\cdots+a_{r}\right)\left(\begin{array}{c}
q+i-2 \\
i-1
\end{array}\right)\left(\begin{array}{c}
r-i+p \\
p-1
\end{array}\right) \\
& =\left(a_{1}+\cdots+a_{r}\right)\left(\begin{array}{c}
p+r-1 \\
r
\end{array}\right) \\
& +\sum_{i=1}^{r-1}\left(a_{i+1}+\cdots+a_{r}\right)\left(\begin{array}{c}
p+r-i-1 \\
r-i
\end{array}\right)\left(\begin{array}{c}
q+i-1 \\
i
\end{array}\right) \text {. }
\end{aligned}
$$


As a direct consequence of Proposition 2.1, we obtain the following.

Corollary 2.2. Let $(R, \mathfrak{m})$ be a DVR. Then, for an arbitrary $R$ module $C$ of finite length, the function $\Lambda(p, q)$ associated to the module $C$ coincides with a polynomial function. Moreover, we have the formula

$$
e^{j}(C)=\ell_{R}\left(R / \operatorname{Fitt}_{j}(C)\right)=e\left(R / \operatorname{Fitt}_{j}(C)\right)
$$

for any $j=0,1, \ldots, r-1$.

Remark 2.3. In [7, Theorem 8.1], Kirby and Rees computed the multiplicities $e^{j}(C)$ in the case where $C$ is a module of finite length and $R$ is a DVR (see also [8, Proposition 4.1]). Our results give more detailed information about the function $\Lambda(p, q)$.

The case where the set of ideals $Q_{1}, Q_{2}, \ldots, Q_{r}$ is not an ascending chain is more complicated. However, the case where $r=2$ can be computed as follows.

Theorem 2.4. Assume $r=2$, and put $I:=Q_{1}+Q_{2}$. Then:

(i) the Buchsbaum-Rim polynomial is

$$
\Lambda(p, q)=\left(a_{1}+a_{2}\right)\left(\begin{array}{c}
p+1 \\
2
\end{array}\right)+e(R / I)\left(\begin{array}{l}
p \\
1
\end{array}\right)\left(\begin{array}{l}
q \\
1
\end{array}\right)-e_{1}(I)(p+q)+c
$$

for all $p, q \gg 0$, where $e_{1}(I)$ denotes the first Hilbert coefficient of $I$ and $c$ is a constant. In particular, we have that

$$
\left\{\begin{array}{l}
e^{0}(C)=\ell_{R}\left(R / \operatorname{Fitt}_{0}(C)\right)=\ell_{R}\left(R / Q_{1} Q_{2}\right) \\
e^{1}(C)=e\left(R / \operatorname{Fitt}_{1}(C)\right)=e(R / I) \\
e^{2}(C)=0
\end{array}\right.
$$

(ii) The function $\Lambda(p, q)$ coincides with a polynomial function if and only if the equality $\ell_{R}(R / I)=e(R / I)-e_{1}(I)$ holds true. When this is the case,

$$
\Lambda(p, q)=\left(a_{1}+a_{2}\right)\left(\begin{array}{c}
p+1 \\
2
\end{array}\right)+e(R / I)\left(\begin{array}{l}
p \\
1
\end{array}\right)\left(\begin{array}{l}
q \\
1
\end{array}\right)-e_{1}(I)(p+q)+e_{1}(I)
$$

for all $p, q \geq 0$. 
(iii) The function $\Lambda(p, q)$ coincides with the following simple polynomial function

$$
\Lambda(p, q)=\left(a_{1}+a_{2}\right)\left(\begin{array}{c}
p+1 \\
2
\end{array}\right)+e(R / I)\left(\begin{array}{l}
p \\
1
\end{array}\right)\left(\begin{array}{l}
q \\
1
\end{array}\right)
$$

if and only if there is an inclusion between $Q_{1}$ and $Q_{2}$.

Before detailing the proof, we will state a lemma which is an explicit description of the function $\Lambda(p, q)$. In order to do this, we will introduce some notation. Let $p, q \geq 0$, and let $\ell=\left(\ell_{1}, \ell_{2}\right) \in \mathbb{Z}_{\geq 0}^{2}$ be such that $|\ell|=p+q$. Let $\delta=\delta(\ell)$ be the number of elements of the set $\Delta=\Delta(\ell)=\left\{\ell_{i} \mid \ell_{i}<p\right\}$.

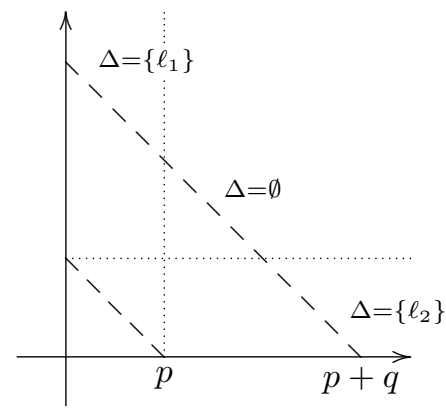

$p \leq q$

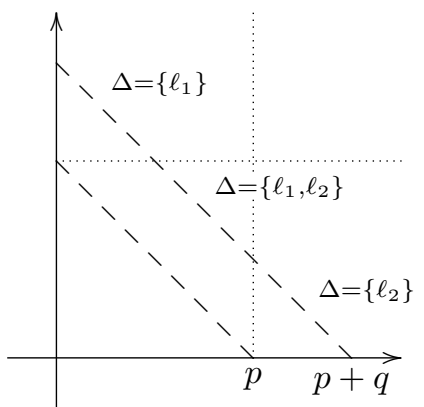

$p>q$.

Let $h_{n}=\ell_{R}\left(R / I^{n}\right)$ be the Hilbert-Samuel function of the ideal $I$. With this notation, we have the following.

\section{Lemma 2.5 .}

(i)

$$
J_{p, q}(\ell)= \begin{cases}I^{p} & \text { if } \delta=0 \\ Q_{j}^{p-\ell_{i}} I^{\ell_{i}} & \text { if } \delta=1, \Delta=\left\{\ell_{i}\right\}, i \neq j, \\ Q_{1}^{p-\ell_{2}} Q_{2}^{p-\ell_{1}} I^{q} & \text { if } \delta=2 .\end{cases}
$$


(ii)

$$
\ell_{R}\left(R / J_{p, q}(\ell)\right)=\left\{\begin{array}{lc}
h_{p} & \text { if } \delta=0, \\
a_{j}\left(p-\ell_{i}\right)+h_{\ell_{i}} & \text { if } \delta=1, \Delta=\left\{\ell_{i}\right\}, \\
a_{1}\left(p-\ell_{2}\right)+a_{2}\left(p-\ell_{1}\right)+h_{q} & \text { if } \delta=2,
\end{array}\right.
$$

$\Lambda(p, q)= \begin{cases}\left(a_{1}+a_{2}\right)\left(\begin{array}{c}p+1 \\ 2\end{array}\right)+2\left(h_{1}+\cdots+h_{p-1}\right)+(q-p+1) h_{p} & \text { if } p \leq q, \\ \left(a_{1}+a_{2}\right)\left(\begin{array}{c}p+1 \\ 2\end{array}\right)+2\left(h_{1}+\cdots+h_{q}\right)+(p-q-1) h_{q} & \text { if } p>q .\end{cases}$

Proof. Assertion (i) is easy and implies assertion (ii). Assertion (iii) follows from assertion (ii). Indeed, if $p \leq q$, then $0 \leq \delta \leq 1$, and we have that

$$
\begin{aligned}
\Lambda(p, q)= & \sum_{\substack{\ell_{1}+\ell_{2}=p+q \\
\ell_{1}, \ell_{2} \geq 0 \\
p-1}} \ell_{R}\left(R / J_{p, q}\left(\ell_{1}, \ell_{2}\right)\right) \\
= & \sum_{\ell_{1}=0}^{p-1}\left(a_{2}\left(p-\ell_{1}\right)+h_{\ell_{1}}\right)+\sum_{\ell_{1}=p}^{q} h_{p}+\sum_{\ell_{1}=q+1}^{p+q}\left(a_{1}\left(p-\ell_{2}\right)+h_{\ell_{2}}\right) \\
= & \sum_{\ell_{1}=0}^{p-1}\left(a_{2}\left(p-\ell_{1}\right)+h_{\ell_{1}}\right)+(q-p+1) h_{p}+\sum_{\ell_{2}=0}^{p-1}\left(a_{1}\left(p-\ell_{2}\right)+h_{\ell_{2}}\right) \\
= & \left(a_{1}+a_{2}\right)(1+2+\cdots+p)+2\left(h_{1}+\cdots+h_{p-1}\right)+(q-p+1) h_{p} \\
= & \left(a_{1}+a_{2}\right)\left(\begin{array}{c}
p+1 \\
2
\end{array}\right)+2\left(h_{1}+\cdots+h_{p-1}\right)+(q-p+1) h_{p} .
\end{aligned}
$$

The case where $p>q$ is similar.

Proof of Theorem 2.4. Let $p_{0}$ be the postulation number of $I$, that is, $h_{p}=e(R / I) p-e_{1}(I)$ for all $p>p_{0}$ and $h_{p_{0}} \neq e(R / I) p_{0}-e_{1}(I)$. To compute the Buchsbaum-Rim polynomial, we may assume that $p_{0}<p \leq q$. Then, by Lemma 2.5 (iii),

$$
\begin{aligned}
\Lambda(p, q)= & \left(a_{1}+a_{2}\right)\left(\begin{array}{c}
p+1 \\
2
\end{array}\right) \\
& +2\left(h_{1}+\cdots+h_{p_{0}}+h_{p_{0}+1}+\cdots+h_{p-1}\right)+(q-p+1) h_{p}
\end{aligned}
$$




$$
=\left(a_{1}+a_{2}\right)\left(\begin{array}{c}
p+1 \\
2
\end{array}\right)+e(R / I)\left(\begin{array}{l}
p \\
1
\end{array}\right)\left(\begin{array}{l}
q \\
1
\end{array}\right)-e_{1}(I)(p+q)+c,
$$

where $c=2\left(h_{1}+\cdots+h_{p_{0}}\right)-e(R / I)\left(p_{0}+1\right) p_{0}+e_{1}(I)\left(2 p_{0}+1\right)$. This proves assertion (i).

Suppose that the function $\Lambda(p, q)$ coincides with the polynomial function. Then, by substituting $p=1$ in the polynomial, $\Lambda(1, q)=$ $\left(e(R / I)-e_{1}(I)\right) q+\left(a_{1}+a_{2}-e_{1}(I)+c\right)$ for any $q \geq 0$. On the other hand, by Lemma 2.5 (iii), $\Lambda(1, q)=h_{1} q+\left(a_{1}+a_{2}\right)$ for any $q \geq 1$. By comparing the coefficients of $q$, we have $h_{1}=e(R / I)-e_{1}(I)$.

Conversely, suppose that $h_{1}=e(R / I)-e_{1}(I)$. Then it is known by [11, Theorem 1.5 and Corollary 1.6] that the Hilbert-Samuel function $h_{n}$ coincides with the polynomial function for all $n>0$. Hence, the function $\Lambda(p, q)$ also coincides with the polynomial function with the following form

$$
\Lambda(p, q)=\left(a_{1}+a_{2}\right)\left(\begin{array}{c}
p+1 \\
2
\end{array}\right)+e(R / I)\left(\begin{array}{l}
p \\
1
\end{array}\right)\left(\begin{array}{l}
q \\
1
\end{array}\right)-e_{1}(I)(p+q)+e_{1}(I)
$$

by Lemma 2.5 (iii). Thus, we have assertion (ii).

For assertion (iii), if the function $\Lambda(p, q)$ coincides with the following simple polynomial function

$$
\Lambda(p, q)=\left(a_{1}+a_{2}\right)\left(\begin{array}{c}
p+1 \\
2
\end{array}\right)+e(R / I)\left(\begin{array}{l}
p \\
1
\end{array}\right)\left(\begin{array}{l}
q \\
1
\end{array}\right),
$$

then $e_{1}(I)=0$ and $h_{1}=e(R / I)$. This implies that $I$ is a parameter ideal for $R$, and hence, $Q_{1} \subseteq Q_{2}$ or $Q_{2} \subseteq Q_{1}$. The other implication follows from Proposition 2.1.

Consequently, there exists the case where the Buchsbaum-Rim function $\Lambda(p, q)$ does not coincide with a polynomial function even if the ring $R$ is Cohen-Macaulay and the module has a parameter matrix. This should be contrasted with a result due to Brennan, Ulrich and Vasconcelos [1, Theorem 3.4] on the classical Buchsbaum-Rim function $\lambda(p)=\Lambda(p, 0)$ associated to a parameter matrix.

\section{REFERENCES}

1. J. Brennan, B. Ulrich and W.V. Vasconcelos, The Buchsbaum-Rim polynomial of a module, J. Algebra 241 (2001), 379-392. 
2. W. Bruns and U. Vetter, Length formulas for the local cohomology of exterior powers, Math. Z. 191 (1986), 145-158.

3. D.A. Buchsbaum and D.S. Rim, A generalized Koszul complex, II, Depth and multiplicity, Trans. Amer. Math. Soc. 111 (1964), 197-224.

4. R. Callejas-Bedregal and V.H. Jorge Pérez, Mixed multiplicities and the minimal number of generator of modules, J. Pure Appl. Alg. 214 (2010), 16421653.

5. F. Hayasaka and E. Hyry, On the Buchsbaum-Rim function of a parameter module, J. Algebra 327 (2011), 307-315.

6. D. Kirby, On the Buchsbaum-Rim multiplicity associated with a matrix, J. Lond. Math. Soc. 32 (1985), 57-61.

7. D. Kirby and D. Rees, Multiplicities in graded rings, I, The general theory, Contemp. Math. 159, American Mathematical Society, Providence, RI, 1994.

8. , Multiplicities in graded rings, II, Integral equivalence and the Buchsbaum-Rim multiplicity, Math. Proc. Cambr. Philos. Soc. 119 (1996), 425445.

9. S. Kleiman and A. Thorup, A geometric theory of the Buchsbaum-Rim multiplicity, J. Algebra 167 (1994), 168-231.

10. , Mixed Buchsbaum-Rim multiplicities, Amer. J. Math. 118 (1996), $529-569$.

11. J. Lipman, Stable ideals and Arf rings, Amer. J. Math. 93 (1971), 649-685.

Department of Mathematics, Asahikawa Campus, Hokkaido University of Education, Hokumon-cho 9-Chome, Asahikawa-shi, Hokkaido, 070-8621, JAPAN

Email address: hayasaka.futoshi@a.hokkyodai.ac.jp 